\title{
Short and long-term quality of life outcomes in children after surgery for Hirschsprung Disease or Anorectal Malformations: A systematic review and meta-analysis
}

Study team:

Dr. Ahmed Nasr

Division of Surgery, CHEO

401 Smyth Rd, Ottawa, Ontario

K1H 8L1

ANasr@cheo.on.ca

Research Coordinator:

Irina Oltean

401 Smyth Rd, Ottawa, Ontario

K1H 8L1

613-737-7600 ext. 3475

IOltean@cheo.on.ca

Student:

Emily Beveridge

EmilyBeveridge@cmail.carleton.ca

\section{Background Information and Scientific Rationale}

\subsection{Background Information}

Hirschprung's disease (HD) and anorectal malformations (ARM) are congenital intestinal anomalies, typically manifesting during early infancy and characterized by the narrowing and aganglionosis of the distal colon and rectum contributing to symptoms of severe constipation and intestinal obstruction (1-3). HD and ARM affect 1 in 5000 live births with a slight male preponderance (i.e., male-to-female sex ratio of 4:1)(4-6). Current surgical approaches for HD and ARM include the resection of most or the entire aganglionic segment, via traditionally developed surgical approaches such as the Swenson, Duhamel, and Soave procedures as well as laparoscopic approaches and the transanal endorectal pull-through (7). These surgical techniques have improved the results of children with HD by decreasing operation time, blood loss, length of hospital stay, and frequency of postoperative complications at three years after surgery (8-10). Despite these short-term advantages, bowel dysfunction (e.g., constipation, fecal incontinence) and enterocolitis after these surgical procedures persists long-term, and can consequently affect the psychosocial health of children with HD or ARM into adulthood (11-14).

Quality of Life (QoL) is a construct aimed at ameliorating the understanding of physical and psychological sequalae of parents, family members, and primary caretakers, of children afflicted gastrointestinal illnesses (15). Results pertaining to the social adjustment of children with ARM are mixed with certain studies reporting difficulties forming relationships (16), elevated behaviour problems (17), no psychological maladjustment (18), or the presence of emotional disturbance among children experiencing frequent soiling accidents (19). Into older age, the major determinant of QoL remains fecal incontinence (20).

Presently, there is paucity of evidence examining quality of life in children with HD and ARM prioritizing dimensions other than physical functioning, such as psychosocial functioning in a precise manner. Although one systematic review examined postoperative complications, 


\section{Short and long-term quality of life outcomes in children after surgery for Hirschsprung Disease or Anorectal Malformations: A systematic review and meta-analysis}

symptoms, and gastrointestinal-related quality of life in children over the age of 10 years, a psychosocial element is lacking (21). Moreover, issues of generalizability persist due to heterogeneous cohorts of children with differing bowel pathologies, which we will simplify by aggregating outcomes for children with HD and ARM. Validated QoL questionnaires and standard outcome reports across all domains of QoL are also lacking. We will address this variability by reporting clinical outcomes by QoL instrument type so that the scoring system and thresholds considered are consistent. As such, our aim was to develop a recent systematic review of existent literature to narratively and quantitatively summarize the physical and social functioning of children affected by HD and ARM to address these limitations (5).

\section{Rationale}

Understanding the quality of life of children affected by HD and ARM will help clinical practitioners, specifically surgeons, create tailored and comprehensive protocols for managing these unpleasant diseases while recognising the physical, social, and emotional toll they may inflict on families. Future recommendations are to incorporate familial perspectives using a family-centred care approach (22).

\subsection{Potential Risks and Benefits}

There are no significant risks associated with this study apart from the risk to confidentiality, which will be protected by our compliance with all CHEO privacy policies.

\section{Study Objectives}

\section{We have designed a systematic review to:}

1. Examine the prevalence of bowel obstruction post-surgery in children aged 0-18 years with anorectal malformations (ARM) and Hirschprung disease (HD);

2. Examine the prevalence of psychosocial problems (as defined by author) in children with anorectal malformations (ARM) and Hirschprung disease post-surgery (HD);

3. Examine parent and child-reported scores in physical QoL domain after surgery in children with HD or ARM; and

4. Examine parent and child-reported psychosocial QoL score after surgery in children with HD or ARM

\subsection{Methodology:}

Search strategy and inclusion/exclusion criteria:

Our study will identify retrospective and prospective cohort studies, case studies with greater than 5 participants, and randomized-control trials where the quality of life (i.e., physical and/or social functioning) of children aged 0-18 years with either anorectal malformations or Hirschprung's disease is reported by children of appropriate age (as defined by author) or parent proxies. See Appendix 1 for our key search terms. Studies published before 2007 and not written in English will be excluded.

The following databases will be searched on Nov 5, 2019: MEDLINE including Epub Ahead of Print, In-Process \& Other Non-Indexed Citations $(1946$ to October 25,2019$)$ and Embase $(1947$ to 2019 October 25) and the CENTRAL Trials Registry of the Cochrane Collaboration (September 2019 Issue) using the Ovid interface. Searches were limited to English, to journal articles, and articles published since 2006 (Appendix 1). Searches were designed and conducted by librarian experienced in systematic reviews, using a method designed to optimize term selection (23). 


\section{Short and long-term quality of life outcomes in children after surgery for Hirschsprung Disease or Anorectal Malformations: A systematic review and meta-analysis}

Population: Children (0-18 years) diagnosed with HD or ARM and parent proxies. Condition: Physical and pyschosocial functioning reports pre (if reported) and post-surgery at short and long-term follow-up. Surgeries for HD and ARM include: Duhamel, Swenson, endorectal pull-through, laparoscopy-assisted pull-through and open pull through etc. Comparator(s)/control: Children without HD or ARM (are otherwise healthy). Exclusion: Case studies less than 5 participants, literature reviews, systematic reviews, editorials, letters to the editor, conference abstracts, and commentaries.

Primary outcome(s): Prevalence of bowel obstruction and psychosocial problems, and parent and child-reported mean or median scores on physical domain (particularly bowel obstruction) and psychosocial domains. If a global psychosocial score is given, this will be reported.

\subsection{Experimental Plan}

Data Management

Records will be downloaded into Reference Manager for duplicate removal and exported to Covidence for screening. Data abstraction will be performed first using Excel and uploaded to REDCap for study details and methodology. A second stage using piloted spreadsheets will be used to obtain outcome data.

\section{Selection process}

Screening will be performed in two steps (title and abstract, full text) against inclusion criteria by two independent reviewers. Consensus and/or resolution of conflicts will be performed by a third reviewer, where necessary.

\section{Data collection process}

A test set of 50 citations will be used to evaluate the inclusion/exclusion criteria. The test set will be created by a member of the investigative team and verified by a second member. All reviewers who contribute to screening will first perform the test set, and must achieve a sensitivity of $\geq 80 \%$ before they are given access to the full set of study records. Reviewers who do not achieve $\geq 80 \%$ sensitivity will be provided with additional training and repeat a second test set. If $\geq 80 \%$ sensitivity is achieved on the repeat test set, the reviewer will be given to access to the full set of study records.

Data will be abstracted onto Excel and REDCap forms by one independent reviewer and verified by the second reviewer. Consensus and/or resolution of conflicts will be performed by a third reviewer, where necessary. The data abstraction form will be piloted by at least two members of the investigative team against a total of five eligible studies.

\section{Data Items and Outcomes}

From all of the studies included in this review, the following information will be obtained: citation details (title of the article and the year of publication); study details including study design; location of the study and its sample size; patient demographics including age, gender, disease diagnosis, average or median follow-up, quality of life questionnaires employed, number of patients with bowel obstruction at short and long-term follow-up, number of patients with any psychosocial problems at short and long-term follow-up, threshold of QoL scales, and parent-reported versus child-reported mean bowel obstruction score and psychosocial score. A global psychosocial score will be extracted that encompasses mental and social domains (if provided). 


\section{Short and long-term quality of life outcomes in children after surgery for Hirschsprung Disease or Anorectal Malformations: A systematic review and meta-analysis}

We will be prioritizing quality of life results post-surgery, regardless of the surgery type, to identify if there is any relationship between disease diagnosis and quality of life after surgery. These findings should aid in guiding future practice and management of children complicated by HD and ARM whilst considering the impact of quality of life on the design of such protocols. Understanding the physical and psychosocial impact of these diseases in the paediatric population can aid in identifying optimal management strategies and consider the perspectives of children and their parents during clinical treatment with a family-centred approach for managing Hirschprung's disease or anorectal malformations.

Data will be sought for the following primary endpoints/outcomes:

1. Mean or median parent and child-reported bowel obstruction score or global physical domain score;

2. Mean or median parent and child-reported psychosocial QoL score or global psychosocial score (if provided);

3. Prevalence of patients with bowel obstruction after surgery at short and long-term follow-up; and

4. Prevalence of child patients with psychosocial problems after surgery at short and longterm follow-up

2.4 Risk of bias (quality) assessment: Observational studies will be assessed using the MINORS 2015 checklist. Researchers will independently perform quality assessment of publications and will compare scores in order to reach a consensus and standardize responses. When consensus cannot be reached, a third researcher will be consulted. Quality assessment will be used to inform interpretations of the data and will help identify studies whose results may be influenced by their study design.

2.5 Strategy for data synthesis: Data to be used will be aggregate or at the level of individual participants. Tables will be used to summarize the general characteristics of the studies included. Depending on the data and the level of variation between studies, a meta-analysis will be used to synthesize data. Count and dichotomous data will be expressed as odds ratios with $95 \%$ confidence intervals, while continuous data will be expressed as a mean or standardized mean difference with $95 \%$ confidence intervals. Forest plots will be utilized to visualize the data. Statistical heterogeneity of the included studies will be assessed using the Isquared test with $95 \%$ confidence intervals, and publication bias will be determined using a funnel plot and Egger's test when possible ( $>10$ included studies). Should there be an insufficient number of studies to allow for meta-analysis, then only a systematic review will be carried out. A subgroup analysis based on surgery type and age may be considered if there is sufficient sample size.

\subsection{Meta-bias(es)}

Two independent researchers will assess the risk of bias to both selective publication and selective non-reporting using GRADE, which evaluates the different sources of reporting bias across separate domains (e.g., selective nonreporting in the 'study limitations' risk of bias domain and bias due to selective publication in the 'publication bias' domain). Publication bias will be determined using a funnel plot and Egger's test when possible ( $>10$ included studies). We will also be assessing the presence of small ('positive') studies with for-profit interest, 
Short and long-term quality of life outcomes in children after surgery for Hirschsprung Disease or Anorectal Malformations: A systematic review and meta-analysis

discrepancy in findings between published and unpublished studies, and whether search strategies were comprehensive or not (24).

\subsection{Confidence in cumulative evidence}

If a meta-analysis will be undertaken, the strength of the body of evidence will be assessed using GRADE. Our evidence will mainly come from non-randomised studies and will be upgraded if there is a large magnitude of effect, evidence of a dose-response effect, and confounders are adjusted for. After the process of upgrading or downgrading is complete, each researcher will rate each outcome as high, moderate, low, or very low (25).

3. Dissemination plans: We intend to publish the review in a relevant peer-reviewed journal such as the Journal of Pediatric Surgery. Results will also be disseminated through conference presentations and academic meetings or research days.

4. Funding sources/sponsors: This study is not funded.

5. Conflicts of interest: There is no conflict of interest to declare. 


\section{Short and long-term quality of life outcomes in children after surgery for Hirschsprung Disease or Anorectal Malformations: A systematic review and meta-analysis}

\section{References}

1. Catto-Smith AG, Trajanovska M, Taylor RG. Long-term continence after surgery for Hirschsprung's disease. J Gastroenterol Hepatol. 2007;22(12):2273-82.

2. Singh SJ, Croaker GDH, Manglick $\mathrm{P}$, Wong $\mathrm{CL}$, Athanasakos $\mathrm{H}$, Elliott $\mathrm{E}$, et al. Hirschsprung's disease: The Australian Paediatric Surveillance Unit's experience. Pediatr Surg Int. 2003;19(4):247-50.

3. Chhabra S, Kenny SE. Hirschsprung's disease. Surg (United Kingdom) [Internet]. 2016;34(12):628-32. Available from: http://dx.doi.org/10.1016/j.mpsur.2016.10.002

4. Bergeron KF, Silversides D, Pilon N. The developmental genetics of Hirschsprung's disease. Clin Genet. 2013;83(1):15-22.

5. Collins L, Collis B, Trajanovska M, Khanal R, Hutson JM, Teague WJ, et al. Quality of life outcomes in children with Hirschsprung disease. J Pediatr Surg [Internet]. 2017;52(12):2006-10. Available from: https://doi.org/10.1016/j.jpedsurg.2017.08.043

6. Espeso L, Coutable A, Flaum V, Rebeuh J, Lavrand F, Podevin G, et al. Persistent Soiling Affects Quality of Life in Children with Hirschsprung's Disease. J Pediatr Gastroenterol Nutr. 2020;70(2):238-42.

7. Torre-Mondragon L, Ortega-Salgado J. Transanal endorectal pull for hirschprung's disease. J Pediatr Surg [Internet]. 1998;33(8):1283-6. Available from: internalpdf://5.89.187.45/De la Torre-Mondragon 1998.pdf

8. Deng X, Wu Y, Zeng L, Zhang J, Zhou J, Qiu R. Comparative Analysis of Modified Laparoscopic Swenson and Laparoscopic Soave Procedure for Short-Segment Hirschsprung Disease in Children. Eur J Pediatr Surg. 2014;25(5):430-4.

9. Gunnarsdóttir A, Larsson LT, Arnbjörnsson E. Transanal endorectal vs. duhamel pullthrough for Hirschsprung's disease. Eur J Pediatr Surg. 2010;20(4):242-6.

10. Lukač M, Antunović SS, Vujović D, Petronić I, Nikolić D, Radlović V, et al. Efikasnost različitih hirurških procedura u lečenju Hiršprungove bolesti kod dece. Vojnosanit Pregl. 2016;73(3):246-50.

11. Chung PHY, Wong KKY, Leung JL, Tam PKH, Chung KLY, Leung MWY, et al. Clinical and manometric evaluations of anorectal function in patients after transanal endorectal pullthrough operation for Hirschsprung's disease: A multicentre study. Surg Pract. 2015;19(3):113-9.

12. Bischoff A, Levitt MA, Bauer C, Jackson L, Holder M, Peña A. Treatment of fecal incontinence with a comprehensive bowel management program. J Pediatr Surg [Internet]. 2009;44(6):1278-84. Available from: http://dx.doi.org/10.1016/j.jpedsurg.2009.02.047

13. Levitt MA, Peña A. Pediatric fecal incontinence: A surgeon's perspective. Pediatr Rev. 2010;31(3):91-101.

14. Engum SA, Grosfeld JL. Long-term results of treatment of Hirschsprung's disease. Semin Pediatr Surg. 2004;13(4):273-85.

15. Frazier TW, Hyland AC, Markowitz LA, Speer LL, Diekroger EA. Psychometric evaluation of the revised child and family quality of life questionnaire (CFQL-2). Res Autism Spectr Disord. 2020;70(November 2019):1-13.

16. Hassink EAM, Rieu PNMA, Brugman ATM, Festen C. Quality of life after operatively corrected high anorectal malformation: A long-term follow-up study of patients aged 18 years and older. J Pediatr Surg. 1994;29(6):773-6.

17. Bai Y, Yuan Z, Wang W, Zhao Y, Wang H, Wang W. Quality of life for children with fecal incontinence after surgically corrected anorectal malformation. J Pediatr Surg. 2000;35(3):462-4. 


\section{Short and long-term quality of life outcomes in children after surgery for Hirschsprung Disease or Anorectal Malformations: A systematic review and meta-analysis}

18. Ludman L, Spitz L. Psychosocial adjustment of children treated for anorectal anomalies. J Pediatr Surg. 1995;30(3):495-9.

19. Ditesheim JA, Templeton JM. Short-term v long-term quality of life in children following repair of high imperforate anus. J Pediatr Surg. 1987;22(7):581-7.

20. Grano C, Aminoff D, Lucidi F, Violani C. Long-term disease-specific quality of life in children and adolescent patients with ARM. J Pediatr Surg [Internet]. 2012;47(7):131722. Available from: http://dx.doi.org/10.1016/j.jpedsurg.2012.01.068

21. Dai $Y$, Deng $Y$, Lin Y, Ouyang R, Li L. Long-term outcomes and quality of life of patients with Hirschsprung disease: A systematic review and meta-analysis. BMC Gastroenterol. 2020;20(1):1-13.

22. Goodrich N, Naslund R, Bossert W, Johnson S, Salcedo S, Lyden L, et al. Learner perceptions of family-centred rounds. Clin Teach. 2020;1-5.

23. Bramer WM, de Jonge GB, Rethlefsen ML, Mast F, Kleijnen J. A systematic approach to searching: An efficient and complete method to develop literature searches. J Med Libr Assoc. 2018;106(4):531-41.

24. Page MJ, McKenzie JE, Higgins JPT. Tools for assessing risk of reporting biases in studies and syntheses of studies: A systematic review. BMJ Open. 2018;8(3):1-16.

25. Meader N, King K, Llewellyn A, Norman G, Brown J, Rodgers M, et al. A checklist designed to aid consistency and reproducibility of GRADE assessments: Development and pilot validation. Syst Rev. 2014;3(1):1-9. 


\section{Short and long-term quality of life outcomes in children after surgery for Hirschsprung Disease or Anorectal Malformations: A systematic review and meta-analysis}

\section{Appendix 1 - Electronic search strategies}

\section{MEDLINE}

1. exp Hirschsprung Disease/ or (hirschsprung* or hirschprung* or hirsprung* or congenital megacolon or aganglionic megacolon or intestinal aganglionosis or colonic aganglionosis or (rectosigmoid adj2 aganglionosis)).ti,ab,kf.

2. Anorectal Malformations/ or (anorectal malformation* or anorectal anomal* or anorectal Atresia* or anal atresia* or anorectal stenos* or imperforate anus).ti,ab,kf.

3. Fecal Incontinence/ or Constipation/ or Defecation/ or Flatulence/ or Diarrhea/ or ((faecal or fecal or feces or faeces or defaecat* or defecat* or stool*) adj2 (problem* or symptom* or function* or incontinen* or continen* or control* or stain* or urgen* or soil* or leak*)).ti,ab,kf. or (diarrhoea or diarrhea or constipate* or flatu* or diaper* or (stool* adj2 (foul or smell or frequen*))).ti,ab,kf.

4. (Baylor Continence Scale or Baylor Social Continence Scale or BCS or HAQL or Holschneider or FII or Kelly score or LSQ or Langemeijer Stool Questionnaire or family impact questionnaire).ti,ab,kf.

5. "Quality of Life"/ or "Outcome Assessment (Health Care)"/ or exp Patient Satisfaction/ or (qol or (quality adj2 life) or patient reported or prom).ti,ab,kf. or questionnaire*.mp. or (well being or wellbeing or satisfaction or quality of life or physical function* or appearance or body image or psychological or sexual or mental or social or psychosocial).ti,ab,kf.

6. (CBCL or $\mathrm{CBCL}^{*}$ or Child Behaviour Checklist or CHQ-PF50 or EuroQoL or FIL or HAQL or HOPES or Hunter Opinions Personal Expectations Scale or mos or pedsql or qqvefca or SF36 or sf-36 or ssp or tacqol or TAIQOL).ti,ab,kf.

7. ( 1 or 2 ) and ( 3 or 4$)$ and ( 5 or 6 ) and (infant* or child* or adolescences*).mp.

8. limit 7 to (english language and yr="2007 - 2020" and journal article)

\section{Embase}

1. exp Hirschsprung Disease/ or (hirschsprung* or hirschprung* or Hirsprung* or congenital megacolon or aganglionic megacolon or intestinal aganglionosis or colonic aganglionosis or (rectosigmoid adj2 aganglionosis)).ti,ab,kw.

2. exp Anorectal Malformation/ or (anorectal malformation* or anorectal anomal* or anorectal Atresia* or anal atresia* or anorectal stenos* or imperforate anus).ti,ab,kw.

3. Feces Incontinence/ or exp Constipation/ or Defecation/ or Defecation Disorder/ or Flatulence/ or exp Diarrhea/ or ((faecal or fecal or feces or faeces or defaecat* or defecat* or stool*) adj2 (problem* or symptom* or function* or incontinen* or continen* or control* or stain* or urgen* or soil* or leak*)).ti,ab,kw. or (diarrhoea or diarrhea or constipate* or flatu* or diaper* or (stool* adj2 (foul or smell or frequen*))).ti,ab,kw. 


\section{Short and long-term quality of life outcomes in children after surgery for Hirschsprung Disease or Anorectal Malformations: A systematic review and meta-analysis}

4. (Baylor Continence Scale or Baylor Social Continence Scale or BCS or HAQL or Holschneider or FII or Kelly score or LSQ or Langemeijer Stool Questionnaire or family impact questionnaire).ti,ab,kw.

5. *"Quality of Life"/ or *Outcome assessment/ or *Patient attitude/ or *Satisfaction/ or exp *Patient Satisfaction/ or (qol or (quality adj2 life) or patient reported or prom).tw. or questionnaire*.mp. or (well being or wellbeing or satisfaction or quality of life or physical function* or appearance or body image or psychological or sexual or mental or social or psychosocial).ti,ab,kw.

6. (CBCL or $\mathrm{CBCL}^{*}$ or Child Behaviour Checklist or $\mathrm{CHQ}-\mathrm{PF} 50$ or EuroQoL or FIL or HAQL or HOPES or Hunter Opinions Personal Expectations Scale or mos or pedsql or qqvefca or SF36 or sf-36 or ssp or tacqol or TAIQOL).ti,ab,kw.

7. ( 1 or 2 ) and ( 3 or 4 ) and ( 5 or 6 ) and (baby* or babies* or newborn* or infan* or neonat* or preschool* or pre-school* or child* or pediatr* or paediatr* or teen* or adolescen*).mp.

8. limit 7 to (Embase)

9. limit 8 to conference abstract

10. 8 not 9

11. limit 10 to (english language and yr="2007 - 2020")

\section{Cochrane Central Trials Registery (CENTRAL)}

1. (hirschsprung* or hirschprung* or Hirsprung* or congenital megacolon or aganglionic megacolon or intestinal aganglionosis or colonic aganglionosis or (rectosigmoid adj2 aganglionosis)).ti,ab,kw.

2. (anorectal malformation* or anorectal anomal* or anorectal Atresia* or anal atresia* or anorectal stenos* or imperforate anus).ti,ab,kw.

3. ( ((faecal or fecal or feces or faeces or defaecat* or defecat* or stool*) adj2 (problem* or symptom* or function* or incontinen* or continen* or control* or stain* or urgen* or soil* or leak*)) or (diarrhoea or diarrhea or constipate* or flatu* or diaper* or (stool* adj2 (foul or smell or frequen*)))).ti,ab,kw.

4. (Baylor Continence Scale or Baylor Social Continence Scale or BCS or HAQL or Holschneider or FII or Kelly score or LSQ or Langemeijer Stool Questionnaire or family impact questionnaire).ti,ab,kw.

5. (qol or (quality adj2 life) or patient reported or prom).tw. or questionnaire*.mp. or (well being or wellbeing or satisfaction or quality of life or physical function* or appearance or body image or psychological or sexual or mental or social or psychosocial).ti,ab,kw.

6. (CBCL or $\mathrm{CBCL}^{*}$ or Child Behaviour Checklist or CHQ-PF50 or EuroQoL or FIL or HAQL or HOPES or Hunter Opinions Personal Expectations Scale or mos or pedsql or qqvefca or SF36 or sf-36 or ssp or tacqol or TAIQOL).ti,ab,kw.

7. ( 1 or 2 ) and ( 3 or 4 ) and ( 5 or 6 ) and (baby* or babies* or newborn* or infan* or neonat* or preschool* or pre-school* or child* or pediatr* or paediatr* or teen* or adolescen*).mp. 
Short and long-term quality of life outcomes in children after surgery for Hirschsprung Disease or Anorectal Malformations: A systematic review and meta-analysis

8. limit 7 to $y r=" 2007-2020$ 